\title{
Engineering trienoic fatty acids into cottonseed oil improves low-temperature seed germination, plant photosynthesis and cotton fibre quality
}

Lihong $\mathrm{Gao}^{1}$, Wei Chen ${ }^{2}$, Xiaoyu Xu ${ }^{3}$, Jing Zhang ${ }^{3}$, Tanoj Singh ${ }^{3}$, Shiming Liu ${ }^{3}$, Dongmei Zhang $^{4}$, Lijun Tian ${ }^{3}$, Adam White ${ }^{3}$, Pushkar Shrestha ${ }^{3}$, Xue-Rong Zhou ${ }^{3}$, Danny

Llewellyn ${ }^{3}$, Allan Green ${ }^{3}$, Surinder Singh ${ }^{3}$, and Qing Liu ${ }^{3}$

${ }^{1}$ Changchun Normal University

${ }^{2}$ Beihua University

${ }^{3} \mathrm{CSIRO}$

${ }^{4}$ Shanghai Landscape Gardening Research Institute

May 5, 2020

\begin{abstract}
Alpha-linolenic acid (ALA, 18:3[?]9,12,15) and $\gamma$-linolenic acid (GLA, 18:3[?]6,9,12) are important trienoic fatty acids which are beneficial for human health in their own right, or as precursors for the biosynthesis of long chain polyunsaturated fatty acids. ALA and GLA in seed oil are synthesized from linoleic acid (LA, 18:2[?]9,12) by the microsomal $\omega-3$ fatty acid desaturase (FAD3) and [?]6 desaturase (D6D), respectively. Cotton (Gossypium hirsutum L.) seed oil composition was modified by transforming with a FAD3 gene from Brassica napus and a D6D gene from Echium plantagineum, resulting in approximately 30\% ALA and $20 \%$ GLA, respectively. The total oil content in transgenic seeds remained unaltered relative to parental seeds. Despite the use of a seed-specific promoter for transgene expression, low levels of GLA and increased levels of ALA were found in non-seed cotton tissues. At low temperature the germinating cottonseeds containing the linolenic acid isomers elongated faster than the untransformed controls. ALA-producing lines also showed higher photosynthetic rates at cooler temperature and better fibre quality compared to both untransformed controls and GLA-producing lines. The oxidative stability of the novel cottonseed oils was assessed, providing guidance for potential food, pharmaceutical and industrial applications of these oils.
\end{abstract}

\section{Hosted file}

Gao_et_al.doc available at https://authorea.com/users/296166/articles/425022-engineeringtrienoic-fatty-acids-into-cottonseed-oil-improves-low-temperature-seed-germinationplant-photosynthesis-and-cotton-fibre-quality

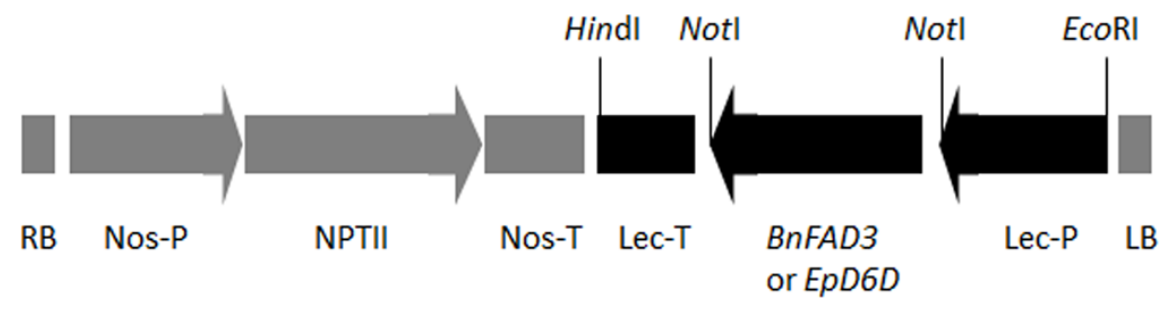



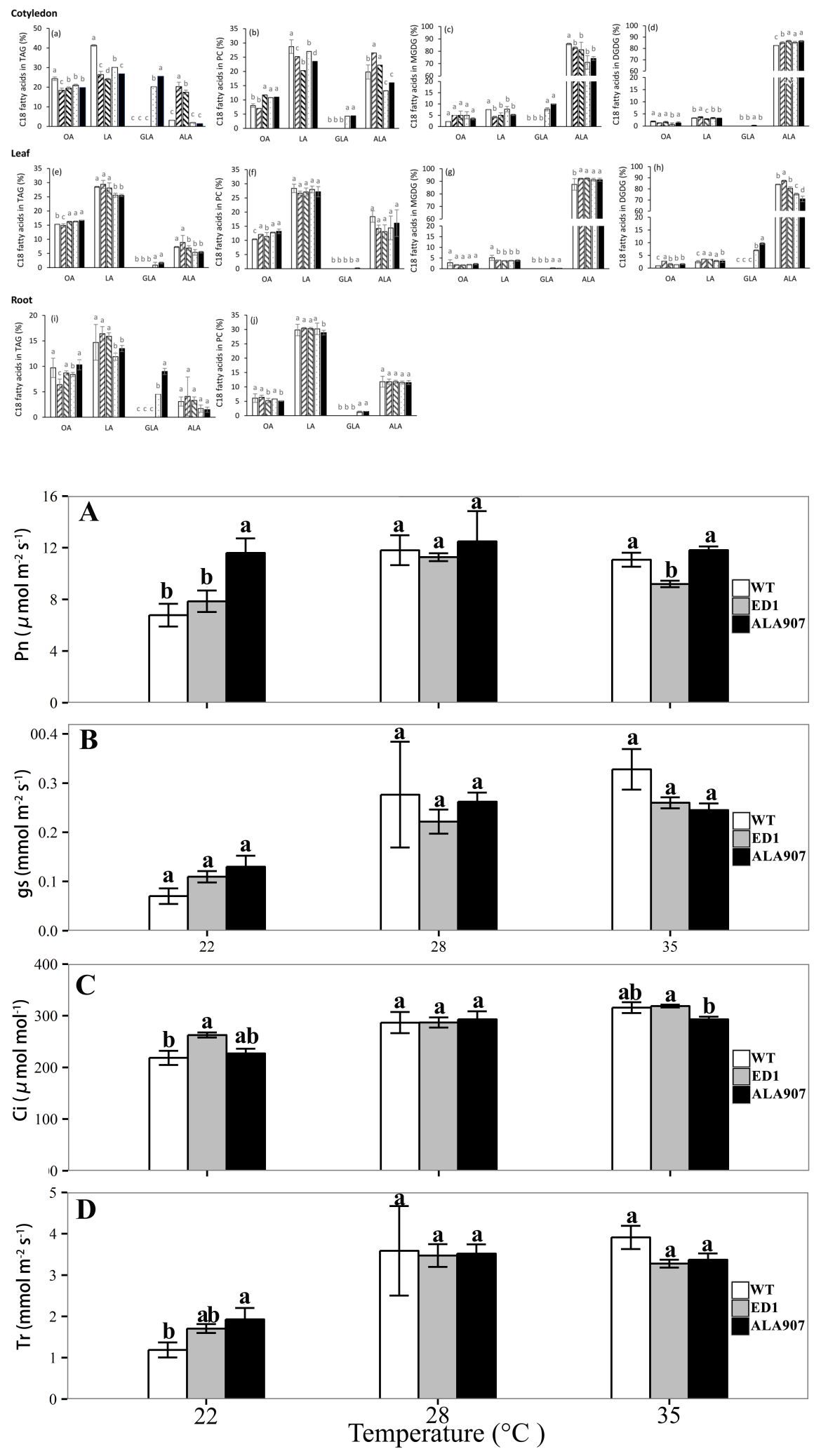

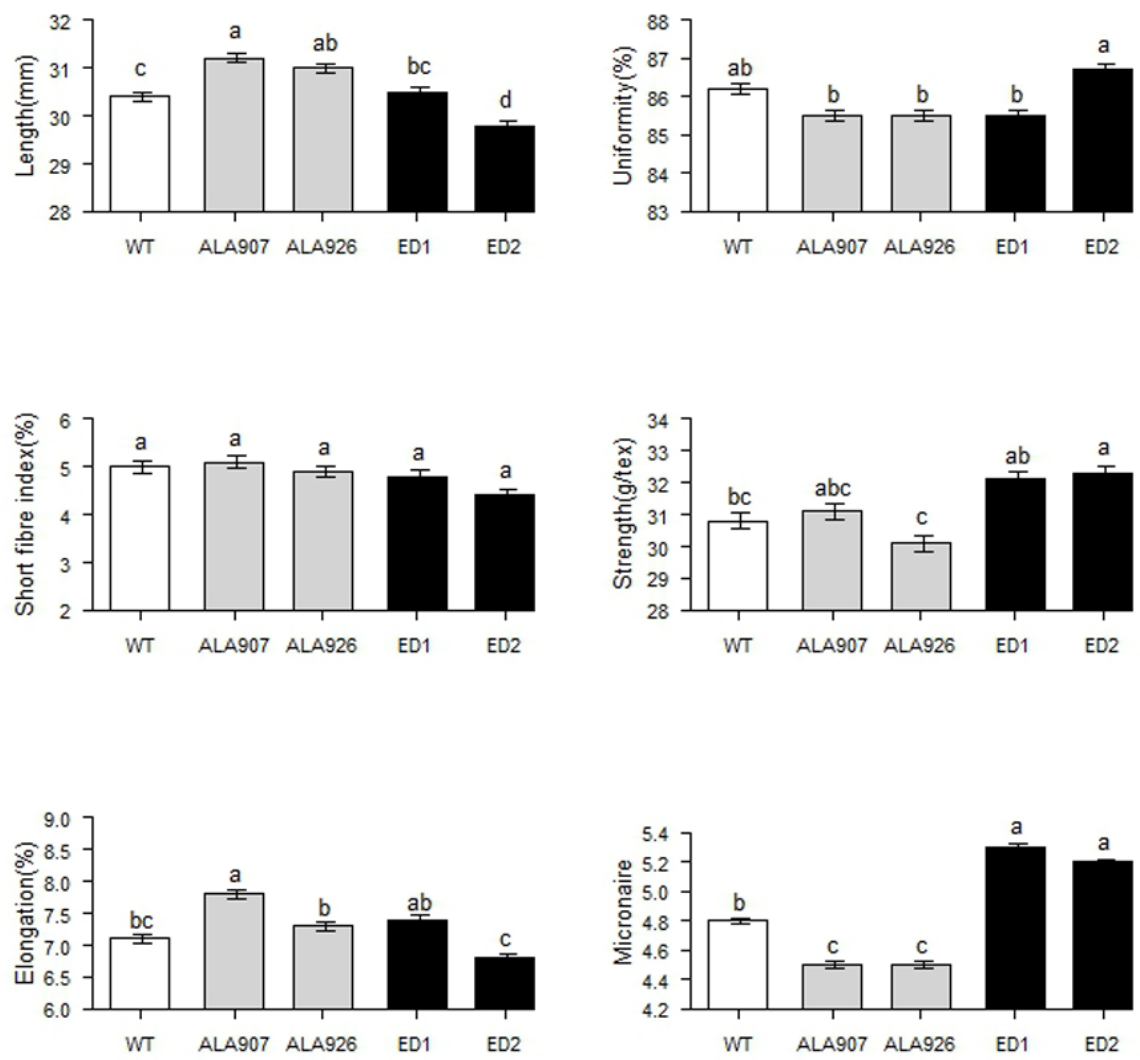
A

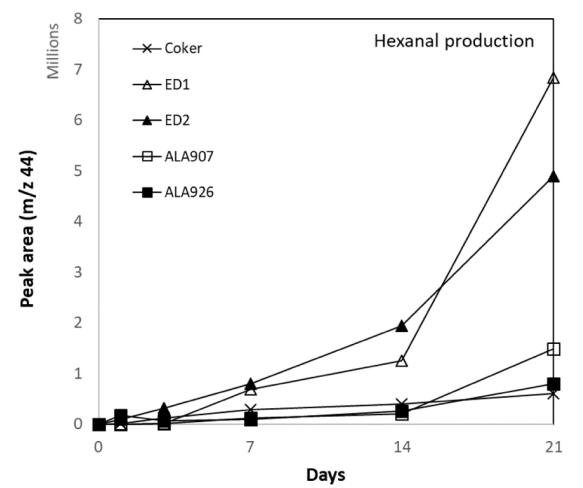

B

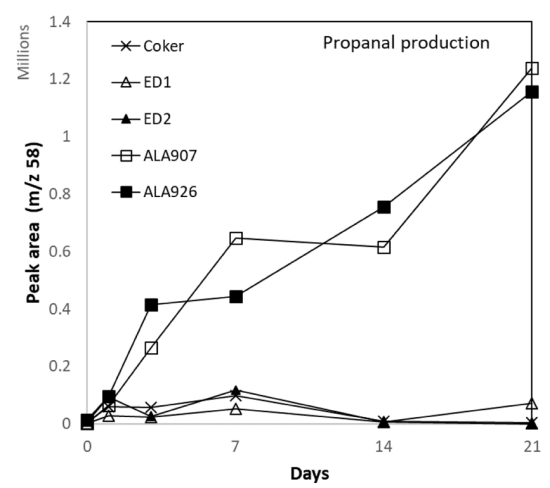

C

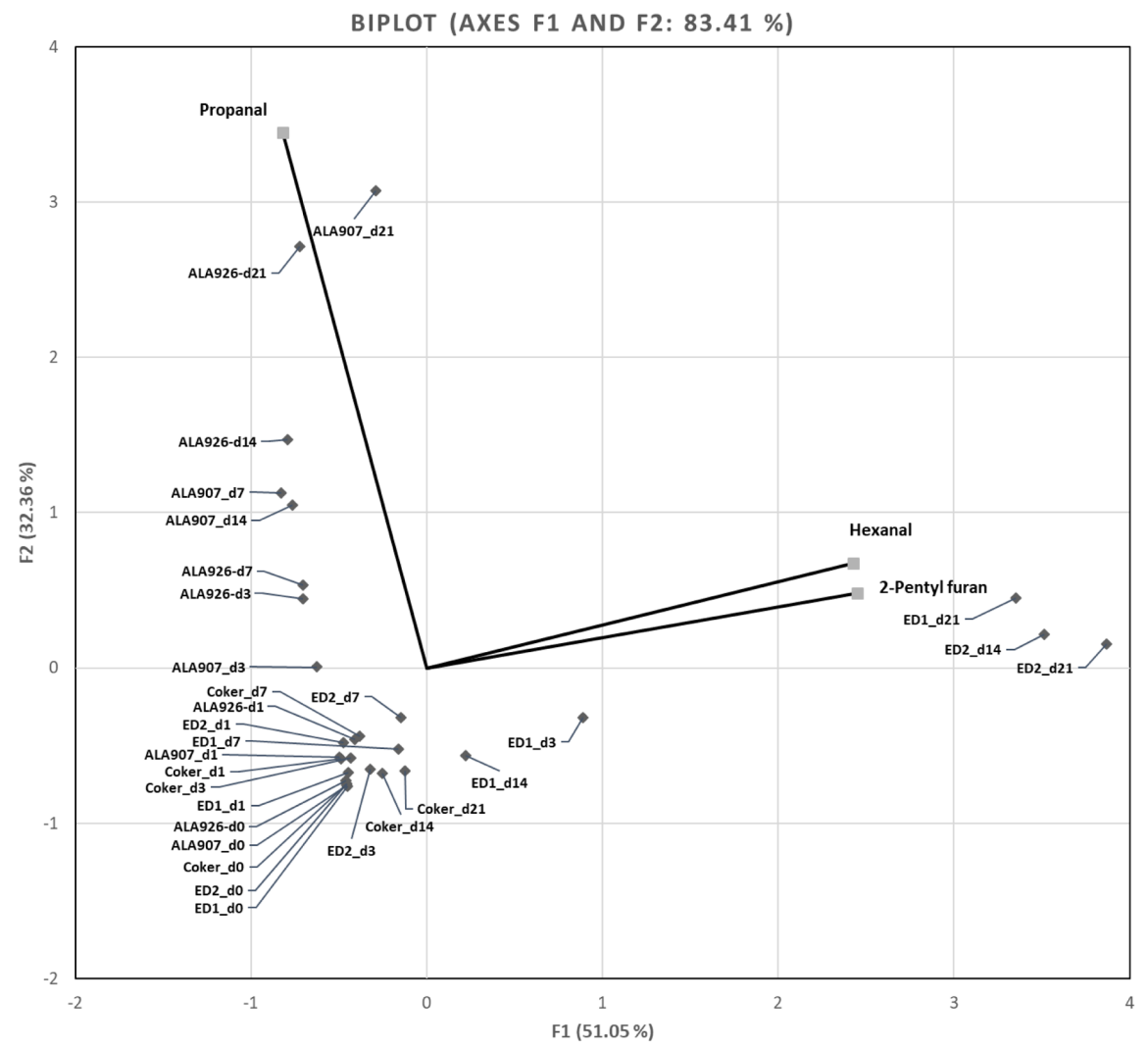

